\title{
Entre histoire et fiction : la Rébellion des Patriotes en roman et en bande dessinée pour la jeunesse québécoise
}

\author{
Amélie Lemieux et Marie-France Guénette
}

La fiction historique se situe à la croisée de deux formes d'écriture : l'une provenant de l'invention de l'auteur, l'autre découlant d'archives et de documents historiques. Le lecteur, quant à lui, «vient au-devant du texte historique [...] avec des attentes [...] qu'on ne lui "raconte pas des histoires" ${ }^{1}$ ». À la différence du texte historique, la fiction historique interpelle le lecteur en lui apprenant des faits réels dans un contexte fictif. Prenant en compte cette définition, nous nous intéressons à deux fictions historiques pour la jeunesse qui retracent un des tournants de l'Histoire du Québec, les Rébellions de 1837-1838. À cet effet, nous nous proposons d'analyser La République assassinée des Patriotes ${ }^{2}$, première bande dessinée fictionnelle qui relate ces évènements, et Nuits rouges ${ }^{3}$, un roman historique pour la jeunesse.

Dans une étude récente sur les défis de la lecture en classe d'histoire, Martel note que «de tous les types de textes, les textes historiques à visée didactique (donc les textes issus des manuels scolaires très utilisés à l'école) sont les plus difficiles à lire pour les élèves ${ }^{4}$ ». Ainsi, nombreux sont les enseignants du secondaire et les chercheurs qui penchent en

1. Paul Ricœur, «L'écriture de l'histoire et la représentation du passé », Annales : Histoire, Sciences Sociales, vol. 55, n 4, 2000, p. 745.

2. Jocelyn Jalette, La République assassinée des Patriotes, Saint-Damien-de-Brandon, Québec, Éditions du soleil de minuit, 2009.

3. Le livre de Daniel Mativat a été finaliste du prix du Gouverneur général en littérature jeunesse de langue française en 2006, Daniel Mativat, Nuits rouges, Saint-Laurent, Québec, Éd. Pierre Tisseyre, coll. « Conquêtes », 2006.

4. Virginie Martel, «Lire en histoire: mieux lire les textes informatifs et s'ouvrir aux ouvres littéraires », Traces: Revue de la Société des professeurs d'histoire du Québec (SPHQ), vol. 51, $\mathrm{n}^{\circ} 1,2013$, p. 18. 
faveur de la fiction historique pour intéresser les élèves à l'Histoire 5 . Notre analyse relève les caractéristiques principales des deux œuvres (le paratexte ${ }^{6}$, les thèmes, et l'enchevêtrement de l'Histoire et de la fiction), tout en les positionnant comme passerelle pour la transmission de la mémoire historique canadienne-française.

Notre corpus combine des éléments fictifs et des faits de l'Histoire canadienne, ce qui constitue une manière dynamique de s'initier à la Rébellion des Patriotes. À titre d'exemple, la bande dessinée reproduit des archives importantes sous forme de lettres imagées, comme la déclaration de l'indépendance du Bas-Canada en ouverture et la lettre d'adieu de Chevalier de Lorimier en annexe. Elle comporte également des éléments paratextuels, dont des notes de bas de page, des cartes géographiques explicatives et des suggestions de lecture qui précisent le contexte spatiotemporel dans lequel se déroule la narration. L'œuvre se veut une bande dessinée à visée didactique, car elle pousse ses lecteurs à réfléchir aux revendications indépendantistes des Canadiens français installés au Québec. Indicatrice de la réflexion proposée aux jeunes, une question apparaît sur la quatrième de couverture : « L'histoire du Québec restera-t-elle toujours une suite d'évènements où les grands espoirs se terminent si souvent en lourdes défaites ${ }^{7}$ ? » La fiction historique participe activement au processus de transmission de la mémoire, et les énoncés des textes font certainement écho aux attentes du Ministère de l'Éducation, du Loisir et du Sport (MELS) relativement aux compétences que doivent acquérir les élèves dans les cours d'histoire au secondaire : « Dans une perspective historique, les élèves sont d'abord amenés à se poser des questions au sujet de l'idée de l'appartenance nationale, aujourd'hui, au Québec, pour s'interroger ensuite sur les revendications et les luttes dans la colonie britannique ${ }^{8} »$.

Le roman jeunesse de Mativat propose, pour sa part, des stratégies didactiques pour accompagner les lecteurs dans leur découverte de faits historiques réels. Il comprend des cartes géographiques, des précisions

5. Voir Martine Jaubert, Sylvie Lalagüe-Dulac et Brigitte Louichon, « Les fictions historiques : Un objet littéraire, éditorial et scolaire qui interroge les frontières », Repères, $\mathrm{n}^{\mathrm{o}} 48,2013$, p. 7-16, et Virginie Martel, «Lire en histoire : mieux lire les textes informatifs et s'ouvrir aux ouvres littéraires », op. cit., p. 18-24.

6 . Dans cet article, nous référons au paratexte tel que défini dans les travaux de Gérard Genette dans Figures III, Paris, Le Seuil, coll. « Poétique», 1972 ; Seuils, Paris, Le Seuil, coll. « Poétique », 1982 ; Palimpsestes : la littérature au second degré, Paris, Le Seuil, coll. « Poétique», 1987.

7. Jocelyn Jalette, La République assassinée des Patriotes, op. cit., quatrième de couverture. 8. Ministère de l'Éducation, du Loisir et du Sport du Québec (MELS), «Programme de formation de l'école québécoise », Histoire et éducation à la citoyenneté, 2011, p. 48. 
factuelles et linguistiques en notes de bas de page, et un lexique. Le roman présente ses apports factuels dans un contexte équilibré, c'està-dire que la "vérité » s'inscrit dans la fiction grâce à des éléments intratextuels et paratextuels. Comme la bande dessinée, le roman incite ses lecteurs à réfléchir sur le rêve de l'indépendance du Québec à l'époque des Patriotes à travers les aventures de Désiré Bourbonnais, un adolescent rebelle et indifférent à la vie politique, qui défend ses valeurs en participant au conflit armé. De telles mises en scène établissent un rapport entre le personnage fictif et les jeunes lecteurs, et stimulent la réflexion sur l'impact de ces évènements. Comme nous le démontrerons dans l'analyse des œuvres, les lecteurs sont ultimement incités à tisser des liens entre la situation sociopolitique des Canadiens français de 1837 et celle dans laquelle ils vivent aujourd'hui.

En donnant vie à des personnages fictifs et historiques dans un cadre vraisemblable, les auteurs de la bande dessinée et du roman brouillent la délimitation entre fiction et réalité. Ainsi, il devient difficile d'établir une ligne de démarcation entre «information attestée $e^{9}$ » et « information fantaisiste ${ }^{10} »$, pour reprendre les mots de Pierre Sève. Cela dit, notre tâche n'est pas de déterminer le degré de fictionnalisation de l'Histoire dans chaque ouvre, mais bien de démontrer dans quelle mesure cette fictionnalisation favorise la compréhension d'une période complexe de l'Histoire du Québec. Comme le précise Robert Bérard, «la littérature ne remplace pas l'histoire, mais elle agit à titre d'instigateur pour le développement de compétences historiques ${ }^{11}$ ». En effet, la fiction historique pour la jeunesse peut permettre d'« apprendre l'histoire, [de] développer la conscience du temps historique [et de] participer au devoir de mémoire ${ }^{12} »$. Ainsi, elle peut à la fois enrichir l'imaginaire du lecteur et l'aider à acquérir les compétences historiques nécessaires pour appréhender l'univers social dans lequel il évolue. Nous émettons l'hypothèse que la fictionnalisation de l'Histoire diminue la lourdeur apparente de la matière, et contribue à accrocher les lecteurs adolescents pour les faire réfléchir aux conséquences politiques et culturelles de cette Rébellion.

9. Pierre Sève, «Lecture des histoires, lecture de l'Histoire. Encyclopédisme et interprétation », Repères, $\mathrm{n}^{\circ}$ 48, 2013, p. 122.

10. Ibid.

11. Notre traduction de « Literature does not substitute itself for history but acts as a catalyst for the development of historical skills » Robert Nicolas Bérard, « Integrating Literature and History: Cultural History in Universities and Secondary Schools », The History Teacher, vol. 16, ${ }^{\circ} 4,1983$, p. 512.

12. Martine Jaubert, Sylvie Lalagüe-Dulac et Brigitte Louichon, « Les fictions historiques : Un objet littéraire, éditorial et scolaire qui interroge les frontières », op. cit., p. 10. 


\section{Démarche}

Nous employons une démarche fondée sur l'analyse successive de La République assassinée des Patriotes et de Nuits rouges. La raison en est double. D'abord, les ouvres racontent l'Histoire des Patriotes par le biais de deux modes, soit la bande dessinée et le roman, lesquels nécessitent une lecture différente (l'un étant multimodal ${ }^{13}$, l'autre monomodal). Ensuite, les deux oeuvres ont certaines similitudes, comme le thème de l'injustice, mais elles affichent des divergences considérables, notamment dans les fonctions du paratexte. Alors que la bande dessinée retrace les moments historiques qui ont ultimement mené, le siècle suivant, à la défaite des séparatistes québécois, le roman jeunesse n'a pas comme objet principal l'avenir du peuple québécois mais bien celui de la quête du protagoniste, apprenti-maçon qui vit les évènements de la Rébellion. En somme, les œuvres explorent la même période historique de manière différente. Ainsi, un croisement systématique des catégories énoncées au moyen d'une analyse comparative forcerait la démarche. Notre analyse successive permet de mettre en lumière les faits saillants de chacune des œuvres, tout en donnant des pistes d'apprentissage de l'Histoire.

Précisément, nous nous attarderons sur l'usage des paratextes, les particularités de la narration, et la fictionnalisation de l'Histoire dans les œuvres susmentionnées. Dans un premier temps, nous décoderons les fonctions du paratexte en présentant des analyses des notes de bas de page, du lexique et des annexes. Ce faisant, nous expliquerons dans quelle mesure les ajouts paratextuels contribuent à resserrer le cadrage historique de l'œuvre, c'est-à-dire comment ils importent des éléments réels dans la fiction. Par ailleurs, nous explorerons les thèmes de l'injustice et de la célébration de la patrie dans la bande dessinée, et ceux de l'incertitude, de l'injustice et de la nostalgie dans le roman. Dans un troisième temps, nous comparerons les récits avec des recensions d'historiens et des archives pour déterminer le degré de fictionnalisation de l'Histoire dans chaque œuvre.

13. À ce sujet, Jean-François Boutin explique : «"Lire"» la bande dessinée [...] c'est donc lire un texte, lire des images, lire un texte/images, lire des sons, lire des gestes, lire une mise en scène », Jean-François Boutin, «De la paralittérature à la littératie médiatique multimodale : Une évolution épistémologique et idéologique du champ de la bande dessinée », dans Monique Lebrun, Nathalie Lacelle, et Jean-François Boutin (sous la direction de), La Littératie médiatique multimodale: De nouvelles approches en lecture-écriture à l'école et hors de l'école, Québec, Presses de l'Université du Québec, 2012, p. 40. 


\section{La République assassinée des Patriotes}

\section{Analyse du paratexte}

\subsection{La couverture : une scène frappante qui contextualise l'histoire}

La page couverture de La République assassinée des Patriotes se présente comme un épisode romancé des Rébellions de 1837-1838. Du côté droit du cadre principal se trouvent les quatre protagonistes, l'un d'eux arborant la traditionnelle ceinture fléchée, « symbole du rêve de construction d'une nation ${ }^{14}$ ". L'homme portant la ceinture fléchée, que nous reconnaitrons dans la diégèse comme la figure de Chevalier de Lorimier ${ }^{15}$, protège trois personnages fictifs: David Gérald, Napoléon Dubrûle, et Amable Galette. Issu d'une famille haïtienne, Gérald, personnage-vedette de Jocelyn Jalette ${ }^{16}$, sert de fervent défenseur des droits des Patriotes. Jalette précise qu'« il n'y a pas de traces réelles qu'un fils d'Haïti a pris une part active au mouvement des Patriotes, mais rien n'empêche historiquement qu'il

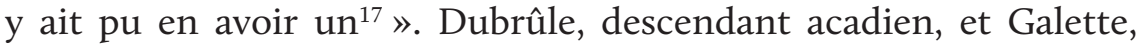
bourgeois de la ville de Québec, agissent comme bras droit de Gérald. Sur la page de couverture, des soldats anglais au regard menaçant les encerclent, mousquets Brown Bess en main. Cette représentation fictive de l'Histoire est expliquée par Jalette : « La page couverture est une allégorie représentant l'arrestation de Chevalier de Lorimier, le 12 novembre 1838, près de la frontière des États-Unis où il tentait de fuir $^{18}$ », un fait historique avéré ${ }^{19}$.

14. François Simard et Louis-Pascal Rousseau, «La ceinture fléchée au carrefour des convoitises des communautés canadiennes-françaises, amérindiennes et métisses du Canada », Revue de la culture matérielle, $\mathrm{n}^{\circ}$ 59, 2004, p. 61.

15. François-Marie-Thomas Chevalier de Lorimier, figure déterminante dans les Rébellions de Patriotes, d'abord comme membre de l'Association des Fils de la Liberté, ensuite comme insurgé contre les dix résolutions de Lord Russell, et finalement comme défenseur des 92 résolutions du mouvement patriote.

16. L’auteur a rédigé une série de bandes dessinées avec Gérald comme protagoniste. Celles-ci sont publiées aux Éditions du Soleil de minuit.

17. Jocelyn Jalette, La République assassinée des Patriotes, op. cit., p. 2.

18. Ibid., page de garde.

19. Nous avons corroboré cette information à l'aide des propos de Francis Back, "Costume : Chevalier de Lorimier ", Cap-aux-Diamants : La revue d'histoire du Québec, $\mathrm{n}^{\circ} 81,2005$, p. 66. 


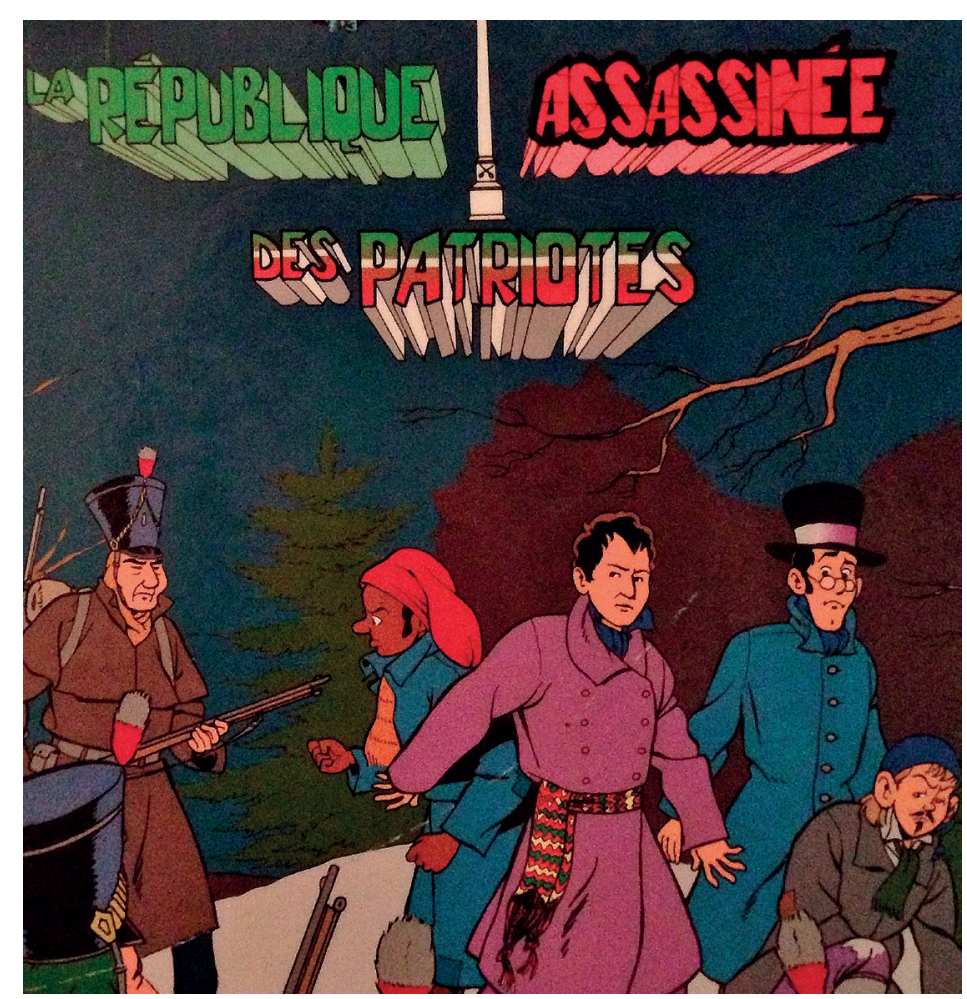

Figure 1 - Jocelyn Jalette, La République assassinée des Patriotes, page couverture, 2009. (C) Éd. du Soleil de minuit. Les couleurs du drapeau des Patriotes présentes dans le titre de la bande-dessinée. La couverture montre la mise-en-scène de l'exil de Chevalier de Lorimier.

Le titre de la bande dessinée arbore des teintes de vert, de rouge et de blanc, couleurs du drapeau des Patriotes. Précisément, « La république » affiche un vert chlorophylle, " assassinée » revêt le rouge sang, et « des patriotes » révèle le vert, le rouge et le blanc du drapeau tricolore. Le rouge d'« assassinée » suggère une double métaphore: il renvoie aux meurtres commis lors des Rébellions, mais aussi à l'assassinat d'une nation indépendantiste canadienne-française. La couleur met en relief le terme "assassinée » et accentue ces métaphores. Le vert de «La république " amplifie aussi le mot auquel il est attribué : cette teinte s'avère un signal symbolisant l'espoir de la conservation d'une nation indépendantiste. Finalement, les couleurs séquentielles du drapeau des Patriotes s'intensifient dans les derniers mots du titre. De 1832 à 1838, ce drapeau a été le symbole du mouvement patriote, dit national ${ }^{20}$, qui

20. Gouvernement du Québec, Justice Québec, « Le fleurdelisé : reflet de notre histoire en Amérique », 2008. 
a lutté en faveur de l'autonomie du Bas-Canada. Ainsi, les paramètres de l'image (le cadrage, la mise en couleur, la composition) contribuent à contextualiser la trame ; c'est-à-dire à produire une « convergence rhétorique $^{21} \gg$ qui cadre l'histoire fictionnalisante des Rébellions.

\subsection{Les fonctions lexicales, historiques, et géographiques des notes de bas de page}

Les renvois en bas de page abondent tout au long de la trame narrative. Ceux-ci servent parfois à expliquer le lexique ou les faits historiques auxquels l'auteur fait allusion, parfois à exprimer un point de vue de l'auteur. Par exemple, Jalette utilise les notes de bas de page pour expliquer certains référents géographiques. À l'aide de la note en page 11, l'auteur explique qu'Ailleboust est aujourd'hui situé à SainteMélanie (dans Lanaudière) et qu'anciennement la ville de Joliette portait le nom de Barthélémy. Ces indications aident le lecteur à localiser les évènements historiques de la narration et à se créer des repères spatiotemporels. À la même page, le terme « Haut-Canada ${ }^{22}$ » fait référence à l'Ontario. Finalement, Jalette emploie « le comté de William-Henry » pour indiquer la ville actuelle de Sorel $^{23}$. Ces référents historico-géographiques rendent la narration riche et instructive.

Les notes offrent également des faits historiques. Par exemple, dans une case, lorsque le bourgeois Galette s'exclame : «J'ai été fier de déposer mes sous à la "Banque du peuple" ! [...] Quelqu'un ose enfin défier le monopole de la "Bank of Montreal"24", la note a pour but d'émettre une explication, d'annoncer une prolepse et de mettre en avant une opinion. Elle se lit comme suit : «Cette institution financière préfigure les caisses populaires fondées par Alphonse et Dorimène Desjardins en 1900 pour affranchir économiquement le peuple. Ottawa s'y opposera, bien sûr !25 » L'auteur donne à l'œuvre un aspect pragmatique et contextualise la narration en présentant des faits historiques sous forme de prolepses externes ${ }^{26}$. L'emploi du mot « préfigure » et l'usage du futur simple en sont des indicateurs. La présence de l'expression «bien

21. Voir à ce sujet Thierry Groensteen, Système de la bande dessinée, Paris, Presses universitaires de France, coll. « Formes sémiotiques », 1999.

22. Nous jugeons cet emploi légèrement imprécis: en 1837-1838, cette région comprend plutôt la partie sud de l'Ontario, au lieu de l'Ontario dans son entièreté, voir à cet effet Archives Canada, Ministère des Services gouvernementaux et des Services aux consommateurs de l'Ontario, dossier « L'Ontario en évolution », 2012.

23. Jocelyn Jalette, La République assassinée des Patriotes, op. cit., p. 17.

24. Ibid., p. 19.

25. Ibid.

26. Gérard Genette, Figures III, op. cit., p. 106-107. 
sûr !» suggère l'opposition flagrante entre la dominance politique canadienne-anglaise et le rassemblement économique des Canadiens français.

L'auteur utilise les notes dans le but de présenter des traductions d'extraits. Certaines expressions apparaissent telles quelles lorsque les anglais s'insurgent : «Hang them! Swine! Rebels! Kill them ${ }^{27}$ ! ». En note de bas de page, l'auteur en offre une traduction française : « Pendez-les! Salauds! Rebelles! Tuez-les!». Très fidèle aux paroles originales, le renvoi traductif a un effet de naturalisation de la narration et de conservation du sens qui privilégie la fidélité à la lettre ${ }^{28}$, c'est-à-dire le respect de l'original. Il permet également au lecteur unilingue francophone de comprendre la vulgarité des mots employés.

Nous avons montré que les notes de bas de page dans La République assassinée des Patriotes assurent quatre fonctions principales: explications géographiques, précisions historiques, émission d'opinion, ou traduction vers le français. Quelle que soit leur fonction dans la séquence narrative, elles jouent un rôle instructif pour le lecteur. Comme catégorie du paratexte, ces renvois forment la «narrativité paratextuelle » que Saint-Gelais définit comme secondaire: "la narrativité de ces notes est en quelque sorte d'emprunt, et toute dépendante de celle du texte principal, auquel elles sont strictement subordonnées ${ }^{29}{ } »$. Nous concédons que ces additions textuelles ne sauraient exister sans la trame narrative première, mais nous jugeons que les notes de Jalette contribuent à exploiter les thèmes du récit. Ceux-ci rappellent la conscience nationale historique québécoise proposée par l'auteur.

\section{La trame narrative de la bande dessinée}

\subsection{Du sentiment d'injustice}

La juxtaposition d'images narratives amène le lecteur à déduire les propositions narratives d'une bande dessinée, même s'il agit comme récepteur de combinaisons images-texte et que celles-ci, de ce fait, freinent son imagination ${ }^{30}$. Dans La République assassinée des Patriotes,

27. Jocelyn Jalette, La République assassinée des Patriotes, op. cit., p. 35.

28. Sur la fidélité à la lettre, voir Antoine Berman, «La traduction et la lettre ou l'auberge du lointain », dans Antoine Berman, Les Tours de Babel, Mauvein, TransEurop-Repress, 1985, p. 31-150.

29. Richard Saint-Gelais, «Enquête sur la narrativité paratextuelle », Protée, vol. 34, $\mathrm{n}^{\circ} 2-3,2006$, p. 81.

30. Voir Barbara Postema, Narrative structure in comics: Making sense of fragments, Rochester, RIT Press, 2013, p. 106. 
les cases accolées racontent les défaites des francophones et le sentiment d'injustice qui persiste depuis la Conquête de 1760. La défaite des Patriotes se présente sous plusieurs formes. D'abord, la page de grand titre atteste bien cette atmosphère: "L'histoire est remplie de ces héros anonymes qui n'ont eu, comme unique récompense, que l'oubli... Souvent, seule la cause qu'ils ont défendue s'est rendue jusqu'à nous. ${ }^{31}$ " Cette proposition introductive donne le ton de la narration : il s'agira d'une bande dessinée retraçant les prouesses héroïques des Patriotes, mais aussi leur défaite aux mains des Anglais.

Lors d'une conversation entre Dubrûle et Gérald au sujet de la domination britannique dans la colonie, ce dernier s'exclame: « [les Anglais] gardent les postes importants pour eux et s'enrichissent à la sueur de nos fronts ${ }^{32} »$. Nous décelons dans ce commentaire des échos du sentiment d'injustice vécu par les Patriotes. Lors du même évènement, Gérald est interrogé par Isabelle, fille de Lord Aylmer, sur sa réticence à «faire partie d'une ${ }^{33}[s i c]$ si noble empire ». Gérald se défend, disant avoir subi une injustice aux côtés de la majorité de la population franco-canadienne: "Personne ne nous a demandé notre avis pour devenir sujets britanniques. En 1760, votre conquête fut notre défaite ${ }^{34}$ ! L Lajout de cette analepse fait écho à une défaite du passé, lorsque la Grande-Bretagne a conquis la Nouvelle-France. Dans la même conversation, Dubrûle s'empresse d'énumérer des évènements de l'Histoire qui ont contribué à développer ce sentiment d'injustice chez les Canadiens français. Notons, par exemple, le salaire attribué aux juges britanniques, les doublés de noms sur les listes salariales, les vols dans le budget militaire, la «composition du conseil législatif» (nommé par le gouverneur au lieu de la Chambre d'Assemblée), et le massacre commandé par Lord Aylmer de trois Patriotes lors de l'élection du 21 mai 1832. L'échange entre personnages fictifs témoigne ainsi de certaines injustices vécues par les Canadiens français.

\subsection{De la célébration de la patrie}

Le récit contient une série d'allusions à la fierté de la patrie canadienne-française. L'auteur emploie des exclamations lancées lors du discours de Chevalier de Lorimier le 22 juin 1837 : «Vive la patrie!

31. Jocelyn Jalette, La République assassinée des Patriotes, op. cit., page de grand titre.

32. Ibid., p. 12.

33. La faute d'orthographe est sans doute un clin d'œil à l'accent anglais, qui a tendance à confondre le genre des déterminants.

34. Ibid., p. 13. 
Oui ! Bravo ${ }^{35}$ ! » À l'occasion d'un rassemblement national, les affiches des citoyens foisonnent: «indépendance» ou encore "liberté nous vaincrons ou mourrons pour elle $\mathrm{el}^{36}$ ! $»$ La foule s'écrie lors des funérailles de Louis-Joseph Papineau «Vive la liberté ! Vive Papineau ${ }^{37}$ ! Dans cette dernière vignette, le cadre exerce une fonction expressive ${ }^{38}$, en ce sens que son tracé irrégulier et sinueux fait écho aux cris célébrant le personnage défunt.
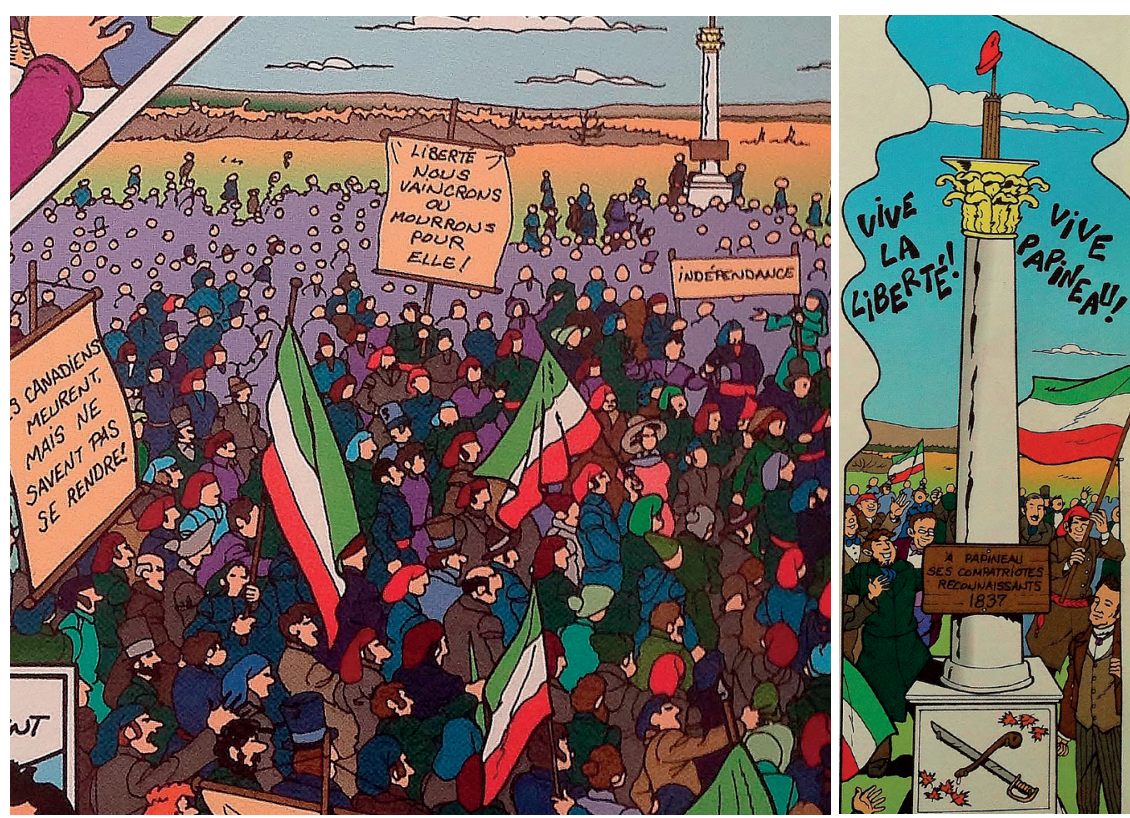

Figure 2 (gauche) - Jocelyn Jalette, La République assassinée des Patriotes, p. 28, 2009. (C) Éd. du Soleil de minuit. Le peuple du Bas-Canada brandit des drapeaux patriotes ainsi que des affiches en faveur de l'indépendance. Figure 3 (droite) - Jocelyn Jalette, La République assassinée des Patriotes, p. 29, 2009. (c) Éd. du Soleil de minuit. Les onomatopées « Vive la Liberté ! » et «Vive Papineau! »sont mises en évidence dans cette case. Des drapeaux patriotes voguent dans le ciel.

Le drapeau tricolore vert, rouge et blanc, symbole des Patriotes, se déploie à plusieurs reprises dans les cases, notamment lors des deux derniers évènements cités. Les citoyens le brandissent également lors de la victoire ${ }^{39}$ des rebelles canadiens-français contre le $32^{\mathrm{e}}$ régiment à

35. Ibid., p. 24.

36. Ibid., p. 28.

37. Ibid., p. 29.

38. Voir les fonctions du cadre cernées par Thierry Groensteen, op. cit.

39. Ibid., p. 33. 
Verchères le 23 novembre 1837, lors de la déclaration solennelle ${ }^{40}$ de Robert Nelson et de l'affrontement du 9 novembre 1838 entre cinq cents Patriotes et un régiment anglais.

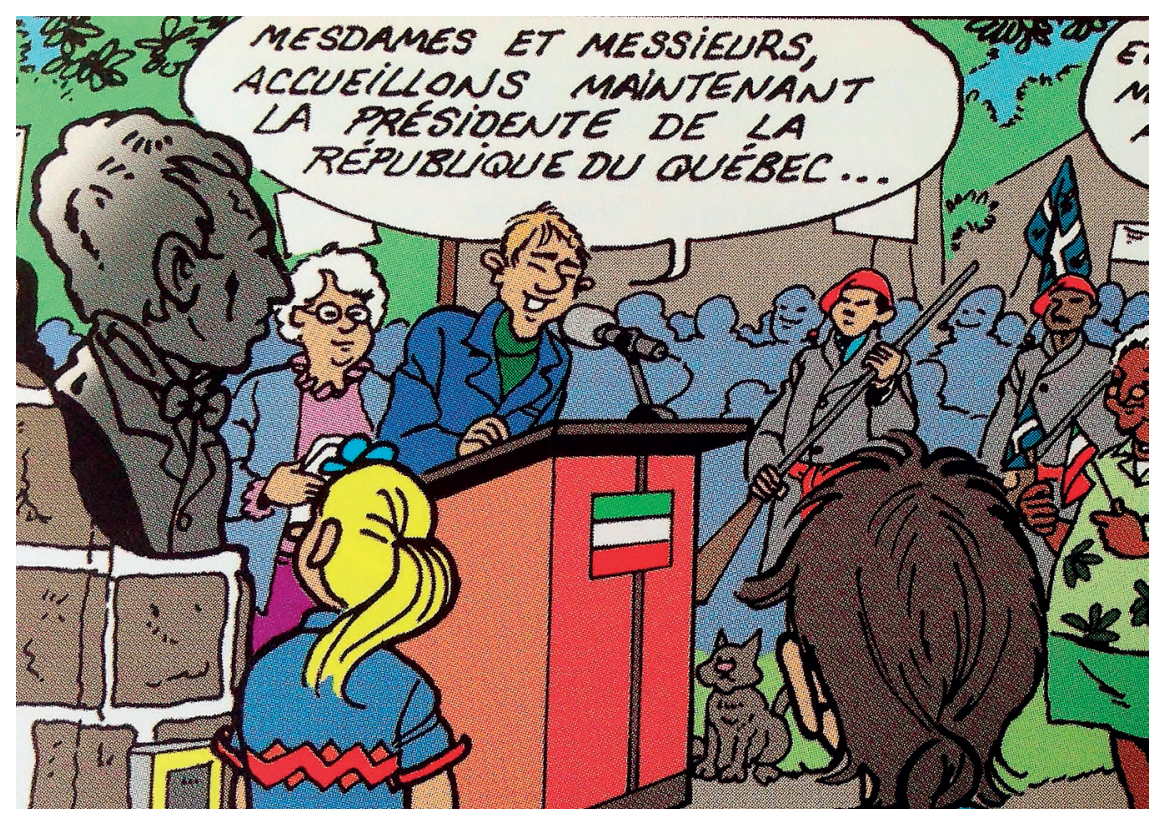

Figure 4 - Jocelyn Jalette, La République assassinée des Patriotes, p. 3, 2009. (C) Éd. du Soleil de minuit. À gauche de la case est située la statue de Chevalier de Lorimier, marqueur d'un événement historique, au centre se trouvent le microphone et les habits contemporains, alors qu'en arrière-plan se trouve le fleurdelisé, donnant le contexte spatio-temporel.

De même, le thème de la célébration de la patrie apparait dès la première case, qui représente environ le quart de la première page. La superficie et le site ${ }^{41}$ de cette case occupent une place de choix dans la bande dessinée. Ainsi, une journée en l'honneur des Patriotes a lieu en début de séquence, et nous pouvons situer la diégèse vers les années 2000 , grâce aux tenues des personnages, à la présence d'un microphone moderne et d'une statue de Chevalier de Lorimier, témoin de son appartenance à l'Histoire du Québec. En arrière-plan, on aperçoit le fleurdelisé, dont la première version fut introduite par Maurice Duplessis en 1948. Ce même drapeau québécois se verra déchu à la toute fin de la narration, lorsque l'auteur explique les statistiques de la défaite souverainiste de 1995. Cet anachronisme reflète par ailleurs un évènement marquant pour les

40. Ibid., p. 39.

41. Voir les notions spatio-topiques de la vignette (forme, superficie, et site) telles que définies par Thierry Groensteen, op. cit. 
sympathisants du mouvement national québécois. Il sert également de repère historique, car il situe la narration dans le cours de l'Histoire de l'émancipation des Canadiens français du Québec.

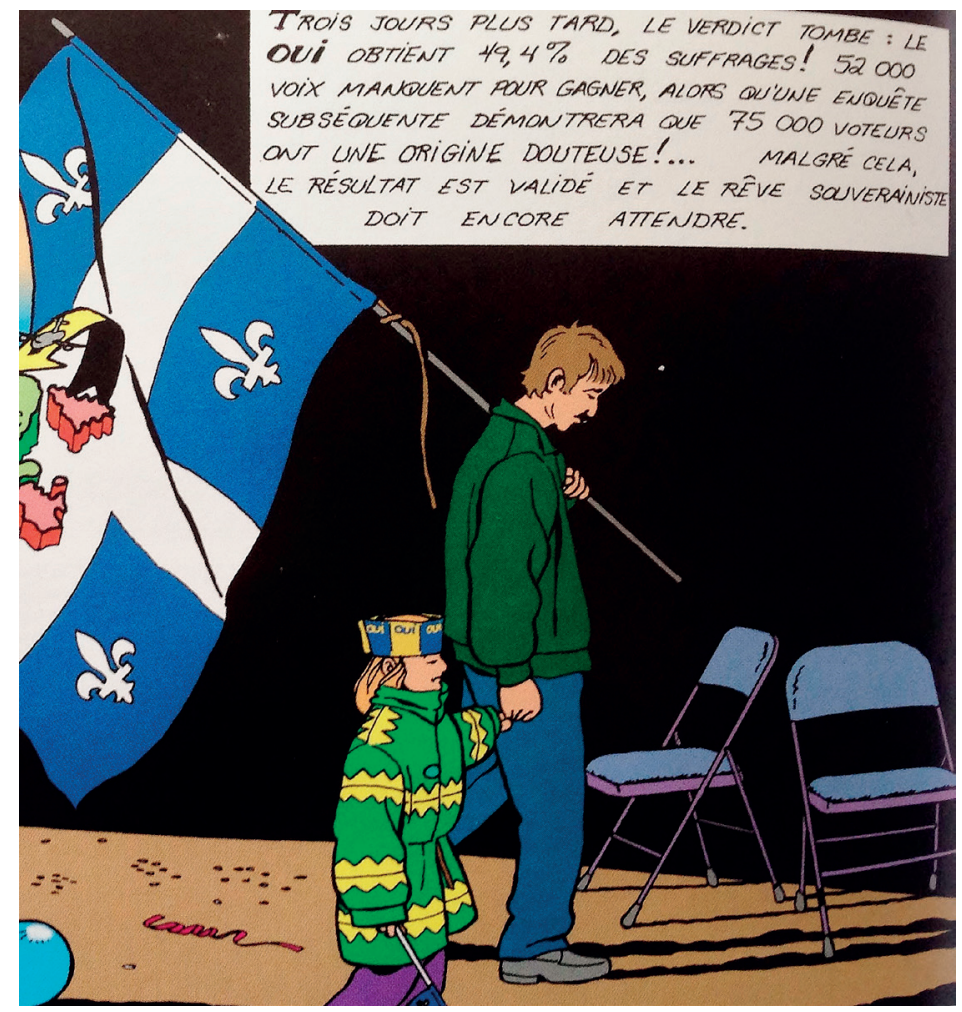

Figure 5 - Jocelyn Jalette, La République assassinée des Patriotes, p. 48, 2009. (C) Éd. du Soleil de minuit. Le drapeau québécois déchu après la défaite référendaire de 1995.

\subsection{La fictionnalisation d'évènements historiques dans La République assassinée des Patriotes}

La fictionnalisation de l'Histoire des Patriotes au Québec se manifeste par la présence du personnage fictif principal David Gérald. Héros d'une série de huit fictions historiques illustrées, ce jeune Québécois d’origine haïtienne veut en apprendre davantage sur ses ancêtres venus d'Haïti pour défendre la cause des Patriotes en 1837-1838. Jalette utilise son 
personnage-vedette pour témoigner de la présence au Québec «des esclaves venus d'Afrique [...] dès la Nouvelle-France ${ }^{42} »$.

L'auteur expose certains liens entre des évènements de l'Histoire du Québec, menant le lecteur à établir des associations plus ou moins directes entre la Rébellion des Patriotes, la première élection du Parti Québécois, et la défaite souverainiste au référendum de 1995. Même si ces évènements sont espacés par plusieurs planches, ils constituent tout de même un réseau, voire un système permettant de se développer sous le thème commun de l'aspiration à l'indépendance qui évolue depuis plusieurs décennies. Il s'agit donc d'un exemple typique de tressage ${ }^{43}$. Si la bande dessinée se veut « un art de la conjonction, de la répétition, de l'enchaînement ${ }^{44}{ }^{\prime}$, alors le travail de reconstitution de la mémoire collective qu'opère Jalette semble réussi à ce niveau. Les thèmes découlant d'évènements historiques infusent l'imaginaire de l'œuvre, qui entremêle fiction, Histoire, et opinions politiques. D'ailleurs, l'œuvre se termine sur ces mots : «Et maintenant? Saurons-nous être dignes de nos ancêtres ${ }^{45}$ ? » Cette fin aux accents patriotiques n'a aucun lien direct avec les batailles des Patriotes du milieu du XIX ${ }^{e}$ siècle. Elle se rapproche davantage du rêve déchu de l'indépendance d'une nation, un thème récurrent dans la diégèse.

Jalette indique que les faits historiques relatés dans sa bande dessinée restent « aussi authentiques que ce que les historiens et les documents consultés ont pu établir ${ }^{46}$ » et que seule «la séquence au manoir d'Ailleboust (p. 11 à 14) [...] a été fabriquée pour résumer les revendications patriotiques ${ }^{47} \gg$. Cette mise en garde fait écho à la subjectivité des historiens. Comme le remarque Charron, « je suis persuadé de l'inévitable subjectivité du discours historique, une subjectivité rigoureuse qui annule tout ce qui n'est pas elle et qui consomme le temps pour en tirer du mérite ou du profit ${ }^{48} »$. Jalette situe son œuvre à la frontière de la fiction et du réel, remettant en cause la véracité même des archives recensées par les historiens.

\section{Ibid., p. 2.}

43. Thierry Groensteen, Système de la bande dessinée, op. cit., p. 174.

44. Ibid., p. 26.

45. Jocelyn Jalette, La République assassinée des Patriotes, op. cit., p. 48.

46. Ibid., page de garde.

47. Ibid.

48. François Charron, « Pour une intelligence rebelle de la conscience (dépaysement) », dans B. Andrés et Z. Bernd (éd.), L'Identitaire et le littéraire dans les Amériques, Québec, Éditions Nota Bene, 1999, p. 214. 
Selon Jalette, la voix du narrateur relate des épisodes historiques réels. La focalisation ${ }^{49}$ est externe ; le narrateur communique des faits historiques de nature diégétique par le texte et ceux de nature mimétique par l'image explicite ${ }^{50}$. Un cartouche en milieu de bande spécifie qu'« en quelques mois, on compta plus de 10000 morts $^{51}$ sur une population totale de 630000 Bas-Canadiens ${ }^{52}$ » à la suite d'une épidémie de choléra de juin à aout 1832. Cette pandémie est racontée comme un fait historique réel dans la diégèse, et aucun personnage fictif n'y est associé. Les cartes géographiques servent de support visuel pour démontrer l'ampleur de la tragédie. De même, lorsque Robert Nelson donne deux discours dans un rassemblement patriote, le cartouche affiche: «Le 28 février 1838, en provenance des États-Unis, des centaines de patriotes franchissent la frontière dans la région de Noyan. C'est là que leur chef, Robert Nelson, procède à la lecture de deux proclamations historiques ${ }^{53}$. »

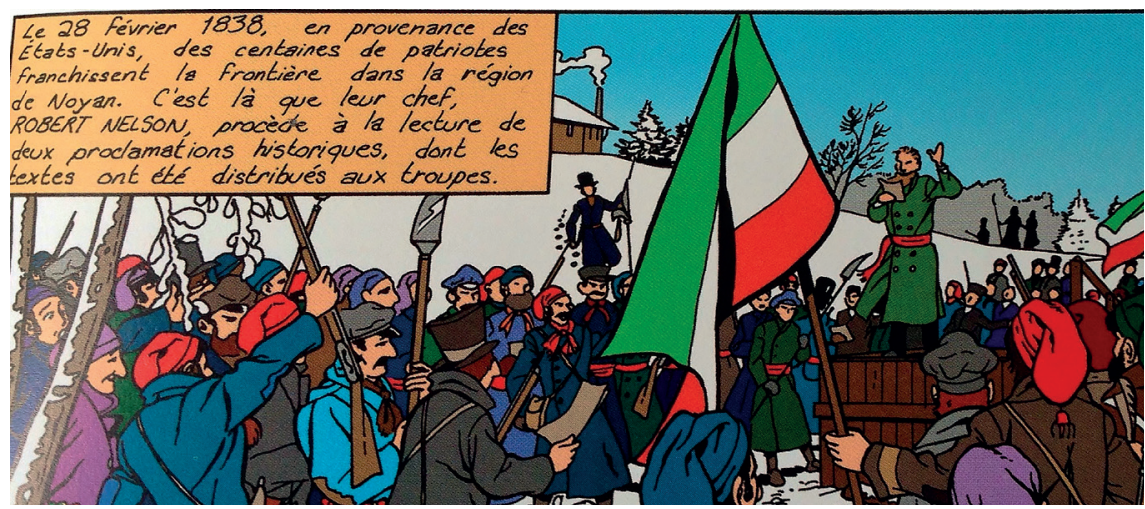

Figure 6 - Jocelyn Jalette, La République assassinée des Patriotes, p. 39, 2009. (C) Éd. du Soleil de minuit. Le discours de Robert Nelson le 28 février 1838.

Certes, les discours sont articulés dans le contexte de la fiction, mais étant donné leur véritable existence dans l'Histoire, il y a anachronisme dans le texte. Bien qu'il s'avère impossible de vérifier si le peuple a trouvé que ces proclamations ont été historiques, il demeure qu'il s'agit de la

49. Gérard Genette, Figures III, op. cit., p. 71-73.

50. Thierry Groensteen, et Ann Miller, 2013, Comics and Narration, Jackson, Presses universitaires du Mississippi, 2013, p. 83.

51. Le nombre exact de victimes reste obscur. Selon Julie Cantoro, « Létat d'alarme de l'époque et le désir de calmer la population faisant que les données recueillies sont incomplètes et inégales $»$, p. 1.

52. Jocelyn Jalette, La République assassinée des Patriotes, op. cit., p. 10.

53. Ibid., p. 39. 
déclaration d'indépendance du Bas-Canada ${ }^{54}$. Jalette fait ainsi appel à la fonction testimoniale du narrateur, c'est-à-dire que le narrateur " prend part à l'histoire qu'il raconte, décrit le rapport qu'il entretient avec elle : rapport affectif, certes, mais aussi bien moral ou intellectuel, qui peut prendre la forme d'un simple témoignage [... ou d'un rapport des] sentiments qu'éveille en lui un tel épisode ${ }^{55} »$.

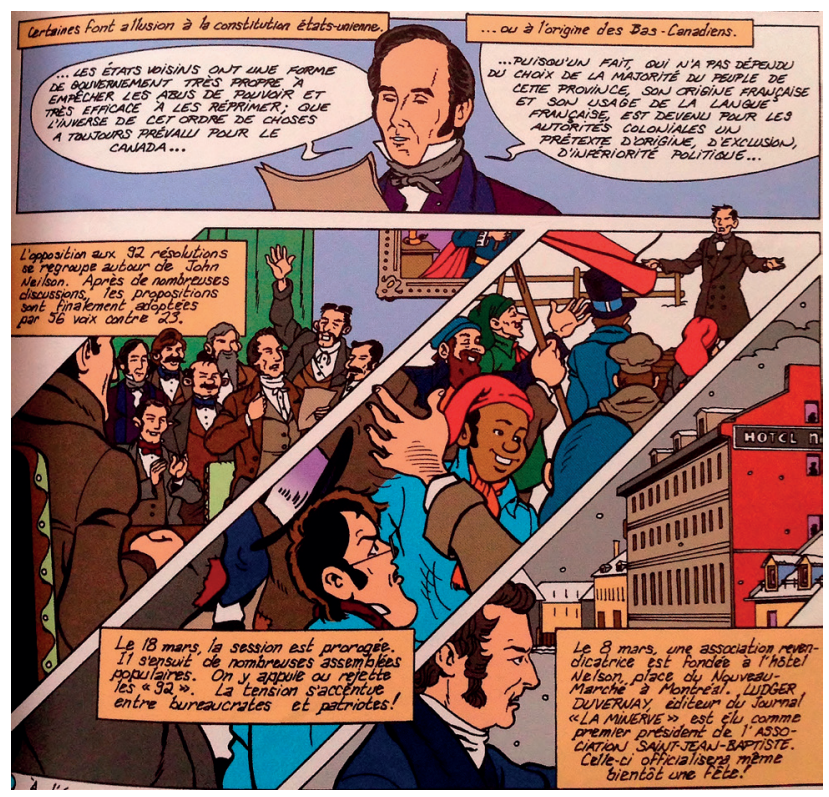

Figure 7 - Jocelyn Jalette, La République assassinée des Patriotes, p. 15, 2009. ( Éd. du Soleil de minuit. N'exprimant aucune parole, David Gérald ne fait qu'une brève apparition en milieu de case.

Présents dans la foule lors du discours parlementaire qu'Elzéar Bédard livre à la Chambre d'Assemblée le 17 février 1834 à propos des 92 résolutions ${ }^{56}$, David Gérald et Amable Galette restent muets. Leur joie, manifestée par un sourire fugace chez Gérald et une expression de stupéfaction chez Galette, ne se voit que par une illustration dans une vignette horizontale. Ici, le narrateur occupe une fonction plus

54. Voir François Labonté, Alias Anthony St. John : Les Patriotes canadiens aux ÉtatsUnis. Décembre 1837-mai 1838, première partie, Saint-Nicolas, Québec, Les Presses de l'Université Laval, 2004, p. 186.

55. Gérard Genette, Figures III, op. cit., p. 262.

56. Les 92 résolutions sont un manifeste politique rédigé par Louis-Joseph Papineau, A.-N. Morin et Elzéar Bédard qui témoigne des « deux dominantes de l'idéologie des Patriotes : le nationalisme et la démocratie », dans Denis Monière, Le Développement des idéologies au Québec des origines à nos jours, Montréal, Éditions Québec-Amérique, 1977, p. 107. 
importante que celle du personnage fictif puisqu'il décrit les faits historiques en cartouches. Personnages-objets, Gérald et Galette témoignent d'une grande passivité lors de ces revendications sur le contrôle des subsides par l'Assemblée et l'administration de la justice au Bas-Canada. Lors de la période de contestation, ils brillent par leur absence : seule l'opposition aux 92 résolutions se démarque. En cartouche, les mots suivants ressortent: "Après de nombreuses discussions, les propositions sont finalement adoptées par 56 voix contre $23^{57}$. »

Cela dit, les trois protagonistes, Gérald, Galette et Dubrûle prennent part à un boycottage de produits d'origine britannique, dont le thé et le rhum, faisant écho au Manifeste de Saint-Ours. Sans que le narrateur nomme ce traité, plusieurs éléments visuels et narratifs y font référence. D’abord, Dubrûle affirme : « Voilà pourquoi notre réponse à cette scélératesse est de priver les occupants britanniques de tout revenu en boudant les produits d'Angleterre ${ }^{58}$ ! $»$ La réplique de Gérald à la case suivante révèle qu'il s'agit bel et bien du Manifeste de Saint-Ours: «D'ailleurs, Napoléon, tu te souviens que nous partons dans quelques jours pour Saint-Ours ${ }^{59}$ ? » Superposée sur ces deux cases se trouve une image d'un baril sur lequel il est écrit « Whisky », et autour duquel on a enserré une ceinture fléchée. Bien que sa superficie soit restreinte, la case occupe un site privilégié et est cernée par un cadre circulaire, qui produit comme un effet d'une loupe. Dérivée de la huitième des 92 résolutions où l'on annonce un boycottage général des produits anglais, la déclaration de Saint-Ours sert d'appel au peuple ${ }^{60}$. En effet, le 20 avril, La Minerve annonce la tenue d'une première grande assemblée à Saint-Ours, dans le comté de Richelieu. Cet évènement se retrouve

57. Jocelyn Jalette, La République assassinée des Patriotes, op. cit., p. 15.

58. Ibid., p. 22.

59. Ibid.

60. Le Manifeste de Saint-Ours « appelle les campagnes à s'organiser et annonçant le boycottage des produits anglais » : "Que nous nous abstiendrons, autant qu'il sera en nous, de consommer les articles importés et particulièrement ceux qui paient des droits plus élevés, tels que le thé, le tabac, les vins, le rhum, etc. Que nous consommerons de préférence les produits manufacturés en ce pays; que nous regarderons comme bien méritant de la patrie quiconque établira des manufactures de soie, de draps, de toiles, soit de sucre, de spiritueux, etc. Que considérant l'acte du commerce comme non avenu, nous regarderons comme très licite le commerce désigné sous le nom de contrebande ; jugerons ce trafic très honorable ; tâcherons de le favoriser de tout notre pouvoir, regardant ceux qui s'y livreront comme méritant bien du pays, et comme infâme quiconque se porterait dénonciateur contre eux », dans Gérard Filteau, "Manifeste de Saint-Ours», Histoire des Patriotes vol. 2: Le nationalisme contre le colonialisme, Montréal, Éditions de l’A.C.-F., 1938, p. 83-87. 
en prolepse dans la bande dessinée lorsque Gérald révèle : « le 7 mai s’y déroulera la première grande assemblée patriote, voyons ${ }^{61}$ !»

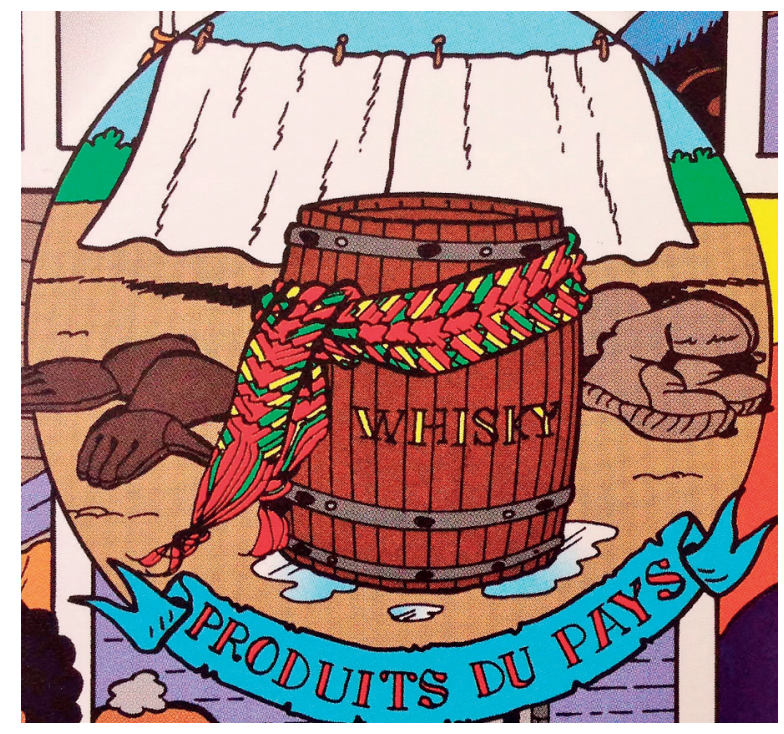

Figure 8 - Jocelyn Jalette, La République assassinée des Patriotes, p. 22, 2009. (C) Éd. du Soleil de minuit. Le baril de whisky autour duquel est attachée la ceinture fléchée. Dans le contexte de la planche, ce dessin se rapporte au Manifeste de Saint-Ours.

La fictionnalisation de l'Histoire dans la bande dessinée porte ainsi une fonction triple : 1 ) représenter une série d'évènements historiques relatant l'Histoire des Patriotes et de l'indépendance du Québec ; 2) se servir de la relation texte-image et des personnages fictifs pour mettre en avant des évènements historiques réels; et 3) accorder un rôle de premier plan au narrateur qui, à l'aide de cartouches imbriqués tout au long de l'œuvre, ponctue la diégèse de faits historiques réels et produit un discours engagé sur ces faits qu'il lie au présent.

\section{Analyse du roman Nuits rouges}

\section{Le genre du roman historique}

Le roman historique, comme toute fiction historique, se définit comme «une narration où les éléments fictifs se mêlent à une proportion plus ou moins forte d'éléments vrais (ou historiques) ${ }^{62} \gg$. Il assure la transmission de la mémoire et initie les lecteurs à des périodes

61. Jocelyn Jalette, La République assassinée des Patriotes, op. cit., p. 22.

62. Gilles Nélod, Panoramas du roman historique, Paris, Éditions Sodi, 1969, p. 22. 
historiques grâce à un travail de recollection d'envergure. En effet, « le propre du roman historique "est d'emprunter son thème à l'histoire" en rendant à l'histoire sa véritable dimension notamment en restituant une conscience historique ${ }^{63} »$. Ainsi, d'après Pouliot, les quatre caractéristiques $^{64}$ principales du roman historique sont: 1) une période mentionnée et datée; 2) des personnages identifiés dans les livres d'histoire ou les manuels d'histoire ; 3) des lieux identifiables sur une carte géographique ou mentionnés dans des manuels d'histoire ; et 4) des récits de personnages fictifs confrontés à des personnages historiques.

Nuits rouges comporte chacune de ces caractéristiques. D’abord, le premier chapitre, "Saint-Eustache, le 16 novembre $1837^{65}$ », annonce à la fois une période et un lieu identifiable. Deuxièmement, l'œuvre comprend des références à des personnages historiques tels Chevalier de Lorimier et Curé Labelle. Troisièmement, l'auteur incorpore une carte de la région de Saint-Eustache en 1837 pour aider le lecteur à visualiser les aspects géographiques du récit. Quant à la confrontation entre la fiction et la réalité historique, le lecteur suit l'impact de la Rébellion des Patriotes sur la vie de l'adolescent Désiré Bourbonnais, un «simple apprenti forgeron ${ }^{66}$ " habitant Saint-Eustache. La maison d'édition et la collection annoncent la visée didactique et littéraire du roman, les Éditions Pierre Tisseyre ayant pour objectif de «publier des auteurs québécois et [de] contribuer au rayonnement de la culture francophone ${ }^{67}$ », et la collection «Conquêtes» ayant une visée didactique qui convient aux lecteurs des premier et deuxième cycles du secondaire. Comme la bande dessinée de Jalette, le roman historique jeunesse de Mativat manifeste une vocation éducative, car il dépeint une période historique complexe en la rendant accessible par la fiction.

\section{Analyse paratextuelle du roman}

Les précisions historiques du paratexte contribuent à transmettre la mémoire du peuple canadien-français dans la littérature jeunesse. Les éléments paratextuels densifient le texte alors que se cumulent cartes géographiques, notes de bas de pages, lexique, bibliographie et témoignages de l'époque des Patriotes. Ceux-ci contribuent à la compréhension, mais perturbent la lecture d'une manière plus exagérée

63. Ibid.

64. Ibid.

65. Daniel Mativat, Nuits rouges, op. cit., p. 11.

66. Ibid., quatrième de couverture.

67. Éditions Pierre Tisseyre, «À propos », Site web des Éditions Pierre Tisseyre, 2014. Page consultée le 25 aout 2015. http://tisseyre.ca/apropos.php 
que dans la bande dessinée, en raison du genre linéaire, conventionnel et textuel du roman. Certains termes restent difficiles à comprendre sans les précisions lexicales, car nombre d'entre eux sont désuets. Les expressions de l'époque ancrent le discours des personnages dans l'espace spatiotemporel. Des astérisques adjacents aux termes nouveaux renvoient à de brèves définitions à la fin du roman. À titre d'exemple, des termes techniques font référence à des objets obsolètes tels " Herminette : Outil à fer plat ou courbe servant à aplanir ou à creuser le bois ${ }^{68} »$ et «Soulier de beu : Botte sans semelle faite de gros cuir ${ }^{69}$ ». Le lexique comprend également des expressions péjoratives telles «Chouayen: loyaliste, partisan du gouverneur ${ }^{70}$ » et « Patroneux: Politicien corrompu. Distributeur de faveurs ${ }^{71} »$. Le champ lexical des insultes indique notamment le degré de tension qui subsistait à l'époque entre les francophones et les anglophones.

Contrairement à la bande dessinée, les notes de bas de page n'occupent que deux fonctions. Certaines apportent des précisions factuelles de nature historique, d'autres donnent des précisions lexicales. Pour reprendre les propos de Richard-Principalli et Fradet, « [u]n certain nombre d'indices paratextuels témoignent de la double lecture attendue, fictionnelle et informative, et courent tout au long [... du roman], depuis la première jusquà la quatrième de couverture ${ }^{72} »$. La vaste gamme d'éléments paratextuels renseigne les jeunes lecteurs en complétant leurs connaissances historiques et culturelles. Les éléments paratextuels deviennent ainsi des dispositifs de médiation qui adaptent la teneur sociologique, historique et politique du roman au lectorat visé.

\section{Faits saillants de la narration}

Les thèmes de l'incertitude, de l'injustice et de la nostalgie s'imposent dans le récit. L'incertitude du protagoniste face à la Rébellion se fait sentir tout au long du roman : "Certes, il n’a jamais aimé les Anglais ni les Loyalistes. Des arrogants et des pisse-vinaigre*. Quant à vouloir faire la révolution son gros bon sens lui dit que cette affaire risque de mener nulle part ${ }^{73} »$. Dans le dénouement, Désiré remet en doute

68. Daniel Mativat, Nuits rouges, op. cit., p. 302.

69. Ibid., p. 304.

70. Ibid., p. 300.

71. Ibid., p. 303.

72. Patricia Richard-Principalli et Marie-Françoise Fradet, « "Histoire d'Histoire”, une collection d'albums historiques composites : Quelles caractéristiques et quels apprentissages en français? », Repères, n 48, 2013, p. 93.

73. Daniel Mativat, Nuits rouges, op. cit., p. 40. L'auteur inscrit une note pour expliquer le sens du mot «Loyaliste», et le terme «pisse-vinaigre » est suivi d'un astérisque 
la cause des Patriotes, car plusieurs combattants l'utilisent comme prétexte pour piller des maisons. Le passage suivant témoigne du scepticisme de Désiré au moment où les Patriotes se préparent à confronter les Anglais :

Le rouquin ne se fait plus guère d'illusions. La grande cause patriotique, il le sait bien, n’attire pas que des rêveurs et des âmes généreuses. Aujourd'hui, combien de ceux qu'il a croisés étaient de vrais patriotes? Combien n'étaient en fait que des profiteurs, des traîne-misère*, des voleurs de poulaillers ou des habitants envieux, trop contents d'avoir l'occasion de pouvoir impunément dépouiller leurs voisins ${ }^{74}$.

Lorsque Désiré se joint à une société secrète qui prépare la deuxième insurrection contre les Anglais, son incertitude resurgit :

Désiré est assailli par le doute. Il a beau être désormais convaincu que combattre aux côtés des Patriotes est une juste et noble cause, il ne peut s'empêcher de trouver que la mascarade à laquelle il vient de se plier est un peu insignifiante sinon franchement ridicule. Sans compter que, si tous les meneurs du nouveau mouvement ont le courage qu'Hippolyte a déjà manifesté face à l'ennemi, l'issue de la lutte est plus qu'incertaine. ${ }^{75}$

Les seules certitudes exprimées par le personnage principal apparaissent à la fin du roman, lorsqu'il revient à Saint-Eustache après son exil en Australie. Désiré souhaite commencer une nouvelle vie au Québec, sur la ferme qu'il a rachetée. Des descriptions de la reconstruction de Saint-Eustache précèdent le passage «La vie est là, toute simple. Inchangée ${ }^{76} »$ indiquent que la vie continue malgré les échecs du passé, et qu'il avait raison de revenir au Québec. Deuxième certitude, en revoyant sa bien-aimée Jeanne, Désiré pense : «Pas de doute, c'est elle $^{77}$ !»

Comme dans la bande dessinée, le thème de l'injustice revient dans la narration; Désiré et les autres Patriotes sont victimes du chaos de leur armée, de leur manque d'éducation, et de leur impuissance face aux Anglais. Travailleur influençable, Désiré hésite à se ranger du côté des Patriotes jusqu'à ce qu'il se fasse menacer de mort : « Mais monsieur, j'ai mon travail, moi.../Le général, qui fait caracoler sa monture sur place, ne lui laisse pas terminer sa phrase./Tu obéis ou je te fais coller contre un

dénotant qu’il est défini dans le lexique. À la p. 303, le lecteur apprend que «pisse-vinaigre » signifie une " personne aigrie qui critique tout ».

74. Ibid., p. 50.

75. Ibid., p. 152.

76. Ibid., p. 295.

77. Ibid., p. 296. 
mur et cribler de plomb ${ }^{78}$. » Les dirigeants des forces rebelles sont peu qualifiés et opportunistes. Le lecteur l'apprend quand les Patriotes se font attaquer par les Anglais et se replient dans l'église de Saint-Eustache. Dans un moment de panique, un des Patriotes demande « Où sont les renforts $^{79}$ ? » Révélant à la fois l'ardeur et la détresse des Patriotes, on lui répond : "Compte pas là-dessus ! Cette ordure de Girod a décampé depuis belle lurette. Dès que ça a commencé à tirailler, il a enfourché son cheval et a filé vers Grand-Brûlé80 . P Plus tard, ce général autoproclamé abandonne la cause et s'exile aux États-Unis. Ces exemples illustrent le fait que, dans le désordre du conflit, l'armée des Patriotes s'est formée rapidement avec peu de ressources.

L'injustice se fait ressentir chez les Patriotes, car la plupart d'entre eux n'ont pas l'éducation nécessaire pour défendre leur désir d'une république. La vie de Désiré représente alors celle des jeunes qui luttent dans ce conflit: "Désiré, qui n'est pas allé à l'école très longtemps, aimerait bien, lui aussi, comme les politiciens, pouvoir justifier ses actes en invoquant la défense de la patrie, l'amour de la liberté ou le droit des peuples à disposer d'eux-mêmes ${ }^{81}$. » Les hommes qui revendiquent les mêmes droits que lui se retrouvent souvent sans voix et se rallient à ceux qui se sont emparés du pouvoir afin de contrer la puissance britannique.

Le thème de la nostalgie se traduit de différentes manières dans le roman. Dès les débuts du mouvement des Patriotes, le personnage principal remarque avec déception que la joie de vivre et la tranquillité de la vie de campagne près de Saint-Eustache se sont estompées. "Chaque habitation qu'il croise, au lieu de lui sembler un repère rassurant, lui apparait comme une sombre menace aussitôt avalée par les ténèbres. Et il finit par comprendre pourquoi il ressent cette sourde angoisse qui lui étreint le cœur. [...] Il n'y a plus personne. Il est seul ${ }^{82}$. » Cette exploitation du thème renvoie à la nostalgie de la vie avant le conflit, celle qui aurait pu continuer si les débats ne s'étaient à ce point intensifiés. La nostalgie partage par ailleurs la tristesse que provoque le projet patriotique déchu.

\section{La fictionnalisation de l'Histoire dans le roman}

Des attributs du cadrage historique de l'œuvre apparaissent tout au long de la narration. L'élément déclencheur du roman, intrinsèquement lié à l'Histoire du Québec, se produit dès le premier chapitre. Le narrateur

\footnotetext{
78. Ibid., p. 39.

79. Ibid., p. 74.

80. Ibid.

81. Ibid., p. 154.

82. Ibid., p. 49.
} 
explique la situation politique et indique pourquoi la langue et la culture divisent les habitants du Bas-Canada. Dans ce passage qui sert de contextualisation diégétique, le lecteur apprend que la vie des Canadiens français de la région de Montréal vient d'être bouleversée. Il s'agit d'un fait historique qui a des répercussions sur la vie des personnages.

Jusque-là, ce n'était rien que des discours et de la politique. Juste de quoi se chicaner un peu entre amis. Mais cet été, les choses ont pris un tour plus inquiétant. À peine la jeune reine Victoria montée sur le trône, voilà que débarque un nouveau gouverneur, Gosford, bien décidé à mater les « rebelles ». Le Parlement est dissout. À Montréal, les fanatiques anglais progouvernement et les « fils de la liberté », militants patriotes, se battent dans les rues à coups de pierre et de gourdins. Comment cela va-t-il finir ? Bien malin qui peut le savoir. En effet, d'après le journal, il semble que la bisbille divise même les Canadiens* entre eux. Pour certains, il faut continuer à se battre en Chambre et paralyser le commerce. Pour d'autres, le temps est venu de prendre les armes. Qui a raison ${ }^{83}$ ?

Le contraste entre la fiction romanesque et la réalité historique transparait dès le premier chapitre. En effet, le lecteur fait la connaissance de quelques habitants de Saint-Eustache, dont un tanneur, un clerc de notaire et un forgeron ; le contact avec l'Histoire se produit lorsque Désiré lit le quotidien à ses confrères. La confrontation des personnages fictifs et des personnages historiques a lieu lorsque les noms d'hommes politiques de l'époque apparaissent dans la narration. À titre d'exemple, le narrateur mentionne Louis-Joseph Papineau, le gouverneur Gosford, de même que le curé de Saint-Eustache, Jacques Paquin. Les noms «Gosford» et « Paquin » sont accompagnés de notes de bas de page pour informer le lecteur qu'il s'agit de personnages historiques réels. Au fil du roman, nous remarquons également les noms l'«abbé Labelle», "Chevalier de Lorimier» et "Émilie Gamelin ». Le Montréal d'aujourd'hui porte plusieurs traces de ces personnages historiques, dont le boulevard Curé-Labelle, l'avenue De Lorimier et la place Émilie Gamelin. La dénomination de ces repères géographiques contextualise l'histoire et témoigne de l'impact de ces derniers sur la culture québécoise.

Le narrateur évoque les contributions de Chevalier de Lorimier au mouvement des Patriotes, sans que celui-ci n'interagisse avec les personnages principaux. Au contraire de la bande dessinée, ses apports sont remis en cause à maintes reprises. Par exemple, le narrateur mentionne ses moments de faiblesse: "Le notaire montréalais De Lorimier, en particulier, cherche à convaincre le docteur 
d'abandonner la partie et de s'enfuir avec eux ${ }^{84} »$. Les personnages secondaires, comme le compagnon de cellule de Désiré, critiquent De Lorimier :

Tu vas finir comme [...] ce toqué de De Lorimier, qui n'arrête pas d'écrire toutes sortes de bêtises pour la postérité : «Vive la liberté ! Vive l'indépendance! Si je meurs sur le gibet, je suis certain que mes frères d'armes prendront soin de mon épouse et de mes gamins », et blablabla! Tu vas voir, s'ils le pendent, comment ses amis prendront soin de sa famille. lls la laisseront crever, tu veux dire ${ }^{85}$ !

Ce passage est une adaptation cynique des vraies lettres écrites par De Lorimier quelques jours avant sa mort. Dans celles-ci, le condamné plaint le sort de son épouse et de ses enfants, à qui il ne laisse aucune sécurité financière. Cependant, il ne demande pas à ses compatriotes de prendre soin d'eux. L'image projetée s'éloigne de celle d'un héros patriotique, alors que l'œuvre de Jalette, les archives du Québec et ses biographes le dépeignent comme un des plus grands héros de l'Histoire de la nation. Dans le passage suivant, tiré d'un livre paru en 1884 et écrit par Laurent-Olivier David (1840-1926), avocat, député et sénateur ayant publié de nombreux livres sur l'Histoire canadienne du xIX ${ }^{e}$ siècle, la mort du notaire De Lorimier est peinte comme une grande perte pour la nation:

De Lorimier avait cessé de vivre. La patrie avait perdu l'un de ses plus nobles, de ses plus généreux enfants, un homme dont le nom vivra et sera honoré aussi longtemps qu'il y aura dans le cœur des Canadiens français le sentiment de l'honneur, du patriotisme et de l'amour de la liberté. Le jour où notre population aura cessé d'admirer ce qu'il y avait de grand, de sublime dans lâme de de Lorimier, elle ne méritera plus de vivre ${ }^{86}$.

Un gouffre se dessine entre les représentations péjoratives de Chevalier de Lorimier dans le roman et celles de son éloge dans les archives et dans la bande dessinée. En raison de la fictionnalisation de l'Histoire dans l'œuvre et de la représentation idéaliste des biographies et archives, il est impossible de déduire de qui relève cet écart. Hypothétiquement, il peut s'agir d'une simple mise en situation pour montrer la discorde entre les Patriotes.

Comme l'exige la fiction historique, les personnages fictifs interagissent avec des éléments historiques véritables et des personnages historiques réels. Ainsi, le roman montre que Désiré est témoin de la

84. Ibid., p. 66.

85. Ibid., p. 194.

86. Laurent-Olivier David, « Les Patriotes de 1837-1838 », La Bibliothèque électronique du Québec, 2002, p. 41. 
pendaison de Lorimier et de ses compatriotes de Beauharnois. Les faits réels influencent également certains aspects de la trame narrative. C'est le cas pour l'évasion de Désiré de la prison du Pied-du-Courant, où le jeune homme parvient à se sauver de la potence. Une note de bas de page à ce sujet spécifie que : « L'évasion de Désiré par le moyen de la folie simulée est inspirée de celle de Félix Poutré, qui devint extrêmement populaire et fit l'objet d'une publication à grand succès : Échappé de la potence, souvenirs d'un prisonnier d'État ${ }^{87}$. » Plusieurs autres situations documentées dans les archives canadiennes s'entremêlent dans la narration, ce qui a pour effet de fictionnaliser l'Histoire tout en rendant la fiction plus réelle.

\section{Conclusion}

Au terme de notre analyse, nous concluons que les discours historiques, politiques et sociaux sous-jacents à la fiction fournissent des renseignements sur cette période décisive de l'Histoire des Canadiens français. Comme le suggèrent Jaubert, Lalagüe-Delac et Louichon, «l'usage de la littérature jeunesse peut paraitre un excellent vecteur pour assumer le devoir de mémoire porté par l'école et la société ${ }^{88} »$. Notre étude de cas démontre la pertinence de faire découvrir la fiction historique aux jeunes lecteurs, au Québec comme ailleurs. Cette transmission de la mémoire rappelle les propos de Ricœur : « c'est en délivrant, par le moyen de l'histoire, les promesses non tenues, voire empêchées et refoulées par le cours ultérieur de l'histoire, qu'un peuple, une nation, une entité culturelle, peuvent accéder à une conception ouverte et vivante de leurs traditions ${ }^{89} »$.

Un discours polyphonique émane des œuvres. La lutte constante contre l'oubli dans laquelle une partie des Souverainistes est engagée suscite encore, en particulier, un sentiment d'injustice et de nostalgie. En raison de la place grandissante de la langue anglaise et de la culture anglophone à l'échelle internationale, les Québécois forment un "peuple en péril dont l'officielle devise - Je me souviens - dit clairement l'attachement à un passé enserré dans la matrice familiale, un passé ajusté au besoin d'affermir un pouvoir ou soutenir une revendication ${ }^{90}{ }^{\prime}$. L'affirmation

87. Daniel Mativat, Nuits rouges, op. cit., p. 144.

88. Martine Jaubert, Sylvie Lalagüe-Dulac et Brigitte Louichon, « Les fictions historiques : Un objet littéraire, éditorial et scolaire qui interroge les frontières », op. cit., p. 9. 89. Paul Ricœur, «La marque du passé », Revue de métaphysique et de morale, n 1 , mars 1998, p. 30-31.

90. François Charron, « Pour une intelligence rebelle de la conscience (dépaysement) », op. cit., p. 214. 
d'une identité francophone demeure un enjeu politique plus de 175 ans après la Rébellion. Comme l'affirme de Certeau: «L'histoire se joue donc là, sur ces bords qui articulent une société avec son passé et l'acte de s'en distinguer; en ces lignes qui tracent la figure d'une actualité en la démarquant de son autre, mais qu'efface ou modifie continuellement le retour du "passé" ${ }^{\prime} »$. Renouveler l'Histoire des Patriotes à l'aide de la fiction devient une ouverture au discours sur l'identité canadiennefrançaise et québécoise.

La nostalgie qui retentit dans les deux œuvres fait écho à la devise du Québec, «Je me souviens ${ }^{92}$ », et rappelle la fragilité d'une Histoire menacée par l'oubli. La reconstitution de l'Histoire à travers la fiction historique pour la jeunesse participe ainsi au devoir de mémoire collective. À ce sujet, Todorov remarque que «la reconstruction collective du passé sert de fondement et de justification à l'image présente de soi ; la mémoire nous procure des gratifications symboliques dont nous avons le plus grand besoin ${ }^{93} »$. En reconstituant des éléments du passé à travers la fiction, nous pouvons comprendre les fondements de notre identité en tournant un regard vers l'avenir. $L a$ République assassinée des Patriotes et Nuits rouges servent d'excellents points de départ pour atteindre ces objectifs.

91. Michel De Certeau, L'Écriture de l'histoire, Paris, Gallimard, 2002 [1975], p. 49-50. 92. Sur l'Histoire de cette devise, voir Gaston Deschênes, « La devise québécoise "Je me souviens" », Encyclopédie du patrimoine culturel de l'Amérique française, 2014.

93. Tzetan Todorov, « La mémoire devant l'histoire », Terrain, n 25, 1995, p. 112. 